\title{
Dependence of the Thomas-Fermi Energy on the Nuclear Coordinates ${ }^{\star}$
}

\author{
Rafael Benguria $\star \star$ \\ The Rockefeller University, New York, NY 10021, USA
}

\begin{abstract}
Let $E(R)$, respectively $e(R)$, denote the total energy, respectively the electronic contribution to the energy, in the Thomas-Fermi theory for a system of two fixed nuclei a distance $R$ apart. We prove that $e(R)$ and $-E(R)$ increase as $R$ does. For the case of $N$ fixed nuclei, we prove the monotonicity of $e$ and $E$ under certain displacements of the coordinates of the nuclei. The analogous result for the electronic contribution to the BornOppenheimer energy is proved.
\end{abstract}

\section{Introduction}

The Thomas-Fermi (TF) theory is defined by the energy functional (in units in which $h^{2}(8 m)^{-1}(3 / \pi)^{2 / 3}=1$ and $|e|=1$, where $e$ and $m$ are the electron charge and mass)

where

$$
\xi(\rho)=\frac{3}{5} \int \rho(x)^{5 / 3} d x-\int V(x) \rho(x) d x+D(\rho, \rho)+U
$$

$$
\begin{gathered}
D(\rho, \rho) \equiv \frac{1}{2} \int \rho(x)|x-y|^{-1} \rho(y) d x d y, \\
V(x)=\sum_{j=1}^{k} z_{j}\left|x-R_{j}\right|^{-1}
\end{gathered}
$$

and

$$
U=\sum_{1 \leqq i<j \leqq k} z_{i} z_{j}\left|R_{i}-R_{j}\right|^{-1} .
$$

Here $z_{1}, \ldots, z_{k} \geqq 0$ are the charges of $k$ fixed nuclei located at $R_{1}, \ldots, R_{k} \cdot \int d x$ is always a three-dimensional integral. $\xi(\rho)$ is defined for electronic densities $\rho(x) \geqq 0$ such that $\int \rho$ and $\int \rho^{5 / 3}$ are finite. The TF energy for $\lambda$ (not necessarily an integer) electrons is defined by

$$
E\left(\lambda ;\left\{R_{i}\right\}\right)=\inf \left\{\xi(\rho) \mid \int \rho=\lambda\right\} .
$$

\footnotetext{
* Research supported by U.S. National Science Foundation under Grant MCS 80-17781
}

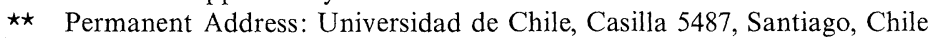


It is known [1] that for $\lambda \leqq Z \equiv \sum_{j=1}^{k} z_{j}$ there is a unique minimizing $\rho$ for (5). It is the unique solution to the TF equation

$$
\rho(x)^{2 / 3}=\max \left(\phi(x)-\phi_{0}, 0\right)
$$

for some $\phi_{0} \geqq 0$ and with

$$
\phi(x) \equiv V(x)-\int \rho(y)|x-y|^{-1} d y .
$$

$-\phi_{0}$ is the chemical potential, i.e.,

$$
\frac{d E}{d \lambda}\left(\lambda ;\left\{R_{i}\right\}\right)=-\phi_{0}
$$

For $\lambda \leqq Z, \phi(x)>0$, all $x . \phi_{0}=0$ if and only if $\lambda=Z$. If $\lambda>Z$, there is no minimizing $\rho$ for (5), and $E\left(\lambda ;\left\{R_{i}\right\}\right)=E\left(Z ;\left\{R_{i}\right\}\right)$ in this case.

Let us denote by

$$
e\left(\lambda ;\left\{R_{i}\right\}\right) \equiv E\left(\lambda ;\left\{R_{i}\right\}\right)-U
$$

the electronic contribution to the TF energy. In this article we study the dependence of both $E\left(\lambda ;\left\{R_{i}\right\}\right)$ and $e\left(\lambda ;\left\{R_{i}\right\}\right)$ on the nuclear coordinates $R_{1}, \ldots, R_{k}$. We will start by indicating some of the previously obtained results concerning this dependence:

(i) It was proven by Teller [2] that for fixed $\lambda \leqq Z$ the TF energy $E\left(\lambda ;\left\{R_{i}\right\}\right)$ is greater than the TF energy for isolated atoms (i.e., the energy when the $R_{i}$ are infinitely far apart). A stronger result has been proven [3] for neutral systems, i.e., for $\lambda=Z$, namely $E\left(\lambda ;\left\{R_{i}\right\}\right)$ decreases under dilation, $R_{i} \rightarrow \ell R_{i}$ $(\ell>1)$, all $1 \leqq i \leqq k$. It has been conjectured [3], but not yet proven, that this should also hold for the subneutral case (i.e., for $\lambda<Z$ ).

(ii) As for the electronic contribution to the energy, it is an elementary consequence of the concavity of $e\left(\lambda ;\left\{R_{i}\right\}\right)$ on the nuclear charges $z_{i}$ that, for fixed $\lambda$ and $z_{i}, 1 \leqq i \leqq k, e\left(\lambda ;\left\{R_{i}\right\}\right)$ always takes its minimum value when all $R_{i}$ are equal ([1], Theorem V.4). (This property also holds for the electronic contribution to the Born-Oppenheimer energy ([4], [5], Theorem 3)). Lieb and Simon $\left([1]\right.$, Theorem V.3) proved that $e\left(\lambda ;\left\{R_{i}\right\}\right)$ is smaller than the corresponding $e$ when the $R_{i}$ are infinitely apart. A stronger result has been conjectured [3] namely, $e\left(\lambda ;\left\{R_{i}\right\}\right)$ should increase under dilations $R_{i} \rightarrow \ell R_{i}$, $\ell \geqq 1$, all $1 \leqq i \leqq k$, for any fixed $\lambda \leqq Z$.

There are other interesting results concerning the dependence of $e$ and $E$ on the nuclear coordinates (see [3], [6]), but they are not relevant for our discussion here. For a review see [7], (Sect. IV).

The main result of this article is

Theorem 1. Fix $z_{i}$, all $1 \leqq i \leqq k$, and $\lambda(\leqq Z)$. Fix $j(1 \leqq j \leqq k)$ and assume that $R_{j}$ is such that

$$
R_{i} \in\left\{x \in \mathbb{R}^{3} \mid\left(x-R_{j}\right) \cdot n \leqq 0\right\}, \quad \text { all } i \neq j
$$


for some fixed $n \in \mathbb{R}^{3}$. Define $E(\lambda ; \alpha) \equiv E\left(\lambda ;\left\{R_{1}, \ldots, R_{j-1}, R_{j}+\alpha n, R_{j+1}, \ldots, R_{k}\right\}\right)$ and $e(\lambda ; \alpha) \equiv e\left(\lambda ;\left\{R_{1}, \ldots, R_{j-1}, R_{j}+\alpha n, R_{j+1}, \ldots, R_{k}\right\}\right)$. Then

(i) $E(\lambda ; \alpha)$ is a monotonic non-increasing function of $\alpha \geqq 0$,

(ii) $e(\lambda ; \alpha)$ is a monotonic non-decreasing function of $\alpha \geqq 0$,

(iii) for fixed $\alpha>0, E(\lambda ; \alpha)-E(\lambda ; 0)$ is non-decreasing in $\lambda$,

(iv) for fixed $\alpha>0, e(\lambda ; \alpha)-e(\lambda ; 0)$ is non-decreasing in $\lambda$. (Hereafter $x \cdot y$ will denote the usual inner product in $\mathbb{R}^{3}$.)

Remarks. (i) The essential content of this Theorem is the following. Let $R_{1}, \ldots, R_{k}$ be given and let $C$ be their convex hull. $C$ has a surface $S$ which is a (possibly degenerate) polyhedron whose vertices are $R_{1}, R_{2}, \ldots, R_{n}$, say. Now consider any displacement of $R_{1}, \ldots, R_{n}$ (and not the other $R$ 's) which has the property that $\left|R_{i}^{\prime}-R_{j}^{\prime}\right| \geqq\left|R_{i}-R_{j}\right|$ for all $i, j$ then $E$ decreases and $e$ increases. Furthermore, if $\lambda_{1}<\lambda_{2}$, then the decrease (increase in $E(e)$ is smaller (larger) for $\lambda_{2}$ than for $\lambda_{1}$. (Here $R_{i}^{\prime}$ denote the new coordinates of the nuclei after the displacement.)

(ii) Note that Theorem 1, (i), (ii) hold for any $\lambda \leqq Z$ and not just for neutral systems.

(iii) For certain nuclear configurations, it follows from Theorem 1, (ii) that $e\left(\lambda ;\left\{R_{i}\right\}\right)$ is not decreased under dilations $R_{i} \rightarrow \ell R_{i}, \ell \geqq 1$, all $i$ (as well as under other displacements of the nuclei). This verifies the (still open) conjecture 4 of [3] for these nuclear configurations.

(iv) In the Born-Oppenheimer approximation, in the case of one electron and $N$ nuclei, the electronic contribution to the energy is monotonic nondecreasing under dilations $R_{i} \rightarrow \ell R_{i},(\ell \geqq 1)$, all $i$ ([5], Theorem 2). We prove below (Sect. 4) that the analog of Theorem 1 (ii) in the Born-Oppenheimer approximation also holds.

In Sect. 2 we introduce, for technical reasons, a regularized TF model. In Sect. 3 we prove Theorem 1 for this regularized TF, and using the convergence of the regularized TF energy to the TF energy, we prove Theorem 1. Finally in Sect. 4 we prove the analog of Theorem 1, (ii) for the Born-Oppenheimer electronic energy.

\section{A Regularized TF Theory}

Let $g$ be a non-negative real-valued function belonging to $C_{0}^{\infty}\left(\mathbb{R}^{+}\right)$and having the properties

(i) $g(r)=0 \quad$ if $r \geqq 1$,

(ii) $4 \pi \int_{0}^{\infty} g(r) r^{2} d r=1$,

(iii) $g(r)$ is a decreasing function of $r$.

For $a>0$, let $g_{a}(x) \equiv a^{-3} g(|x| / a), x \in \mathbb{R}^{3}$. For $0<a<\min \left\{\left|R_{i}-R_{j}\right| \mid 1 \leqq i<j \leqq k\right\}$, define

$$
V_{a}(x)=\left(g_{a} * V\right)(x)
$$


(i.e., $V_{a}$ is the Coulomb potential corresponding to smeared nuclei; we have imposed an upper bound on $a$ so that the smeared nuclei do not overlap). Since $V \in L_{\text {loc }}^{1}, V_{a} \in C^{\infty}\left(\mathbb{R}^{3}\right)$.

Let us define a regularized TF theory by the functional

with

$$
\xi_{a}\left(\rho ;\left\{R_{i}\right\}\right)=\frac{3}{5} \int \rho(x)^{5 / 3} d x-\int V_{a}(x) \rho(x) d x+D(\rho, \rho)+U_{a},
$$

$$
U_{a}=U_{a}\left(\left\{R_{i}\right\}\right) \equiv \sum_{1 \leqq i<j \leqq k} z_{i} z_{j} \iint d y d x g_{a}(x) g_{a}(y)\left|x-R_{i}-y+R_{j}\right|^{-1} .
$$

That is, the functional $\xi_{a}\left(\rho ;\left\{R_{i}\right\}\right)$ is of exactly the same form as the TF functional $\xi(\rho)$, given by $(1)$, the only change being in that we have smeared the singularities of $V(x)$. (Note that we have also changed the constant term $U$ by $U_{a}$ which represents the interaction between the smeared nuclei.)

The minimization problem associated with $\xi_{a}(\rho)$ is contained in the family of variational principles studied in Refs. [1] and [8]. We summarize below those properties of this regularized TF theory that we will need in the sequel:

(i) The problem $\operatorname{Min}\left\{\xi_{a}(\rho) \mid \rho \in L^{1} \cap L^{5 / 3}, \rho(x) \geqq 0, \int \rho(x) d x=\lambda\right\}$, has a unique solution for $0 \leqq \lambda \leqq Z$. For $\lambda>Z$ there is no solution. (That $Z$ is the largest $\lambda$ for which there is a solution follows from [8], Theorem 3 (c), since

$$
\left.\int\left(-\Delta V_{a}\right)=\int\left(-\Delta V_{a}\right)_{+}=\sum_{i=1}^{k} z_{i}=Z .\right)
$$

(ii) The unique minimizing $\rho_{a}$ satisfies the Euler equation

$$
\rho_{a}^{2 / 3}(x)=\max \left(\phi_{a}(x)-\phi_{0}, 0\right)
$$

for some $\phi_{0} \geqq 0$, where

$$
\phi_{a}(x) \equiv V_{a}(x)-\int d y \rho_{a}(y)|x-y|^{-1} .
$$

(iii) $\phi_{a}(x)$ and thus $\rho_{a}(x)$ are continuous everywhere and they go to zero at infinity. (This follows from (14), since $V_{a}(x)$ is continuous everywhere and goes to zero at infinity and so does $\rho_{a} *|x|^{-1}$ (see [1], Theorem II.25) because $\rho_{a} \in L^{5 / 3} \cap L^{1}$ and $|x|^{-1} \in L^{5 / 2}+L^{4}$.)

Let us define the total energy of the regularized TF theory by

$$
E_{a}\left(\lambda ;\left\{R_{i}\right\}\right) \equiv \min \left\{\xi_{a}\left(\rho ;\left\{R_{i}\right\}\right) \mid \int \rho=\lambda\right\}
$$

and the corresponding electronic contribution by

$$
e_{a}\left(\lambda ;\left\{R_{i}\right\}\right) \equiv E_{a}\left(\lambda ;\left\{R_{i}\right\}\right)-U_{a}\left(\left\{R_{i}\right\}\right) .
$$

We are interested in the dependence of $E_{a}$ and $e_{a}$ on the nuclear coordinates; in particular let us keep $R_{2}, \ldots, R_{k}$ fixed and study the change on $E_{a}$ and $e_{a}$ under the shift $R_{1} \rightarrow R_{1}+\alpha n$, with $\alpha \geqq 0, n \in \mathbb{R}^{3}$. Denote by $E_{a}(\lambda ; \alpha) \equiv E_{a}\left(\lambda ;\left\{R_{1}+\alpha n, R_{2}, \ldots, R_{k}\right\}\right)$ and $e_{a}(\lambda ; \alpha) \equiv e_{a}\left(\lambda ;\left\{R_{1}+\alpha n, R_{2}, \ldots, R_{k}\right\}\right)$. Let $V^{\alpha}$ be given by (3) with $R_{1}$ replaced by $R_{1}+\alpha n$ and $V_{a}^{\alpha} \equiv g_{a} * V^{\alpha}$. We have the following 
Theorem 2. (A Feynman-Hellman theorem for regularized TF.) Let $\rho^{\alpha}$ be the unique minimizing $\rho$ for $\xi_{a}\left(\cdot ;\left\{R_{i}\right\}^{\alpha}\right)$ with $\int \rho^{\alpha}=\lambda$. (Here we have used $\left\{R_{i}\right\}^{\alpha}$ to denote $\left\{R_{1}+\alpha n, R_{2}, \ldots, R_{k}\right\}$.) Then,

(i) The functions $\alpha \rightarrow E_{a}(\lambda ; \alpha)$ and $\alpha \rightarrow e_{a}(\lambda ; \alpha)$ are continuously differentiable and

(ii) $\partial e_{a} / \partial \alpha=-\int \partial V_{a}^{\alpha} / \partial \alpha(x) \rho^{\alpha}(x) d x$

$$
=z_{1} \int\left[n \cdot \nabla g_{a}(y)\right] \psi^{\alpha}\left(y+R_{1}+\alpha n\right) d y,
$$

(iii) $\partial E_{a} / \partial \alpha=-z_{1} \int\left[n \cdot \nabla g_{a}(y)\right] \phi^{\alpha}\left(y+R_{1}+\alpha n\right) d y$,

where $\psi^{\alpha} \equiv \rho^{\alpha} *|x|^{-1}$ and $\phi^{\alpha} \equiv V_{a}^{\alpha}-\psi^{\alpha}$.

Proof. Let us first prove that the mapping $\alpha \rightarrow \int \partial V_{a}^{\alpha} / \partial \alpha(x) \rho^{\alpha}(x) d x$ is continuous. We need only prove continuity at $\alpha=0$. Note that $V_{a}^{\alpha} \rightarrow V_{a}$ in $L^{5 / 2}+L^{4}$ (Lemma A.1), and therefore $\rho^{\alpha} \rightarrow \rho^{0}$ in $L^{5 / 3}$ because of Theorem II.15 in [1]. Since $\left\|\rho^{\alpha}\right\|_{1} \leqq \lambda$, we also have $\rho^{\alpha} \rightarrow \rho^{0}$ in (weak) $L^{p}$, any $1<p<5 / 3$. Let $Y^{\alpha} \equiv \partial V_{a}^{\alpha} / \partial \alpha$, we have

$$
\int Y^{\alpha} \rho^{\alpha}-\int Y^{0} \rho^{0}=\int\left(Y^{\alpha} \rho^{\alpha}-Y^{0} \rho^{\alpha}\right)+\int\left(Y^{0} \rho^{\alpha}-Y^{0} \rho^{0}\right) .
$$

The first term in the right side of (18) goes to zero as $\alpha \downarrow 0$ because $Y^{\alpha} \rightarrow Y^{0}$ in $L^{5 / 2}+L^{4}$ and $\rho^{\alpha}$ is bounded in $L^{5 / 3} \cap L^{4 / 3}$. Also $\int Y^{0}\left(\rho^{\alpha}-\rho^{0}\right) \rightarrow 0$, as $\alpha \downarrow 0$, because $Y^{0} \in L^{5 / 2}+L^{4}$ and $\rho^{\alpha} \rightarrow \rho^{0}$ in $L^{5 / 3}$ and $\rho^{\alpha} \rightarrow \rho^{0}$ in (weak) $L^{4 / 3}$, hence $\alpha \rightarrow \int \partial V_{a}^{\alpha} / \partial \alpha(x) \rho^{\alpha}(x) d x$ is continuous.

Now, we prove differentiability of $e_{a}$. Again we need only prove it at $\alpha=0$. For $\alpha>0$,

$$
\begin{aligned}
& \alpha^{-1}\left[e_{a}(\lambda ; \alpha)-e_{a}(\lambda ; 0)\right] \leqq \alpha^{-1}\left\{\left[\xi_{a}\left(\rho^{0} ;\left\{R_{i}\right\}^{\alpha}\right)-U_{a}\left(\left\{R_{i}\right\}^{\alpha}\right)\right]\right. \\
& \left.-\left[\xi_{a}\left(\rho^{0} ;\left\{R_{i}\right\}\right)-U_{a}\left(\left\{R_{i}\right\}\right)\right]\right\}=-\int \alpha^{-1}\left(V_{a}^{\alpha}-V_{a}\right)(x) \rho^{0}(x) d x,
\end{aligned}
$$

by the minimization property of $\rho^{\alpha}$. By the minimization property of $\rho^{0}$

$$
\alpha^{-1}\left[e_{a}(\lambda ; \alpha)-e_{a}(\lambda, 0)\right] \geqq-\int \alpha^{-1}\left(V_{a}^{\alpha}-V_{a}\right)(x) \rho^{\alpha}(x) d x .
$$

Now, $\alpha^{-1}\left[V_{a}^{\alpha}-V_{a}\right] \rightarrow \partial V_{a}^{\alpha} /\left.\partial \alpha\right|_{\alpha=0}$ in $L^{5 / 2}+L^{4}$ (see Lemma A.2 in the Appendix) and $\rho^{0} \in L^{5 / 3} \cap L^{1}$ hence, by Hölder's inequality, the right side of (19) converges to $-\int \partial V_{a}^{\alpha} /\left.\partial \alpha\right|_{\alpha=0}(x) \rho_{0}(x) d x$ as $\alpha \downarrow 0$. Similarly, one can prove that the right side of (20) converges to the same limit as $\alpha \downarrow 0$ and, therefore,

$$
\lim _{\alpha \downarrow 0} \alpha^{-1}\left[e_{a}(\lambda ; \alpha)-e_{a}(\lambda ; 0)\right]=-\left.\int \frac{\partial V_{a}^{\alpha}}{\partial \alpha}\right|_{\alpha=0}(x) \rho^{0}(x) d x .
$$

A similar argument controls $\lim$. Thus $e_{a}(\lambda ; \alpha)$ is continuously differentiable in $\alpha$ and its derivative is given by (15). In order to prove (16), we just compute $\partial V_{a}^{\alpha} / \partial \alpha(x)=z_{1} \int g_{a}(y) n \cdot \nabla_{y}\left|x-y-R_{1}-\alpha n\right|^{-1} d y$, and after introducing this expression in (15), we integrate by parts. Finally, since $U_{a}\left(\left\{R_{i}\right\}^{\alpha}\right)$ is continuously differentiable with respect to $\alpha$ (see Lemma A.3), $\alpha \rightarrow E_{a}(\lambda ; \alpha)$ is continuously differentiable. Moreover, 


$$
\frac{\partial U_{a}\left(\left\{R_{i}\right\}^{\alpha}\right)}{\partial \alpha}=-z_{1} \int\left[n \cdot \nabla g_{a}(y)\right] V_{a}^{\alpha}\left(y+R_{1}+\alpha n\right) d y
$$

and adding the right sides of (16) and (22) we get the right side of (17). This concludes the proof of the theorem.

\section{Proof of Theorem 1}

We will need some geometric preliminaries. For $n$, a unit vector in $\mathbb{R}^{3}$ and $d \geqq 0$, let $P(n, d)$ be the plane

$$
P(n, d) \equiv\left\{x \in \mathbb{R}^{3} \mid x \cdot n=d\right\},
$$

and let $P^{+}(n, d)$ denote the open half-space

$$
P^{+}(n, d) \equiv\left\{x \in \mathbb{R}^{3} \mid x \cdot n<d\right\},
$$

and $P^{-}(n, d)=\mathbb{R}^{3} \backslash\left(P^{+}(n, d) \cup P(n, d)\right)$. For any function $f: \mathbb{R}^{3} \rightarrow \mathbb{R}$ we define

$$
\begin{aligned}
& f^{+}(x)=f(x) \\
& f^{-}(x)=f(x+2(d-x \cdot n) n),
\end{aligned}
$$

for every $x \in P^{+}(n, d) \cup P(n, d)$, i.e., $f^{-}$is just the reflection of $f$ through the plane $P(n, d)$. In order to prove Theorem 1 we will need the following:

Lemma 3. Let $n$ and $d$ be such that $R_{i} \in P^{+}(n, d) \cup P(n, d)$ for all $1 \leqq i \leqq k$ and choose $a<\min \left\{\operatorname{dist}\left(R_{j}, P(n, d)\right) \mid R_{j} \notin P(n, d)\right\}$, (this condition on a insures that the smeared nuclei with $R_{i} \in P^{+}(n, d)$ are supported on $\left.P^{+}(n, d)\right)$. Let $\rho_{a}(x)$ be the unique solution to the regularized TF equation (13) and $\phi_{a}$ the corresponding potential (14). Then

(i) $h_{a}(x) \equiv \phi_{a}^{+}(x)-\phi_{a}^{-}(x)>0$, all $x \in P^{+}(n, d)$

(ii) For each $x \in P^{+}(n, d), h_{a}(x)$ strictly decreases when $\lambda$ increases, and

(iii) $\rho_{a}^{+}(x)-\rho_{a}^{-}(x) \geqq 0$, all $x \in P^{+}(n, d)$.

Proof. (i) Certainly $h_{a}(x)=0$ for $x \in \partial P^{+}(n, d)=P(n, d)$ and at infinity. Let $D=\left\{x \in P^{+} \mid h_{a}(x)<0\right\}$. Since $\phi_{a}$ is continuous everywhere (see Sect. 2$), D$ is open. On $D,-(4 \pi)^{-1} \Delta h_{a} \geqq \rho_{a}^{-}-\rho_{a}^{+}>0$ since, through Eq. (13), $\phi_{a}^{-}>\phi_{a}^{+}$implies $\rho_{a}^{-}>\rho_{a}^{+}$. Thus, $h_{a}$ is superharmonic on $D$, so $D$ is empty. By the strong maximum principle, $h_{a}(x)>0$, in fact, for $x \in P^{+}(n, d)$. (ii) Let $\lambda^{\prime}<\lambda$ with corresponding $h_{a}^{\prime}$ and $h_{a}$. We want to prove that $B=\left\{x \in P^{+} \mid h_{a}(x)-h_{a}^{\prime}(x)>0\right\}$ is empty. Since the $h_{a}$ 's are continuous, $B$ is open and $h_{a}-h_{a}^{\prime}=0$ on $P(n, d)$ and at infinity. We have

with

$$
-(4 \pi)^{-1} \Delta\left(h_{a}-h_{a}^{\prime}\right)=-b_{+}^{3 / 2}+c_{+}^{3 / 2}+p_{+}^{3 / 2}-q_{+}^{3 / 2} \equiv r,
$$

$$
b=\phi_{a}^{+}-\mu, \quad c=\phi_{a}^{-}-\mu, \quad p=\phi_{a}^{+\prime}-\mu^{\prime}, \quad q=\phi_{a}^{-\prime}-\mu^{\prime}, \quad\left(\mu=\phi_{0}^{(\lambda)}, \mu^{\prime}=\phi_{0}^{\left(\lambda^{\prime}\right)}\right) .
$$

By (i) ([1], Theorem V.9), $b>c \geqq q$ and $b \geqq p>q$, for all $x \in P^{+}$. On $B, b+q>c+p$. Thus $r \leqq 0$ on $B$, whence $h_{a}(x)-h_{a}^{\prime}(x)$ is subharmonic on $B$ and therefore $B$ 
is empty. Again, once can prove the stronger result that $h_{a}(x)-h_{a}^{\prime}(x)<0$ for $x \in P^{+}$. Trivially, (i) implies (iii) through the TF equation (13).

Remarks. (i) The proof of Lemma 3, (i) is analogous to the proof of Teller's lemma ([1], Theorem V.8).

(ii) By the same argument once can prove the following two lemmas.

Lemma 4. Let $n$ and $d$ be such that $R_{i} \in P^{+}(n, d)$ for all $1 \leqq i \leqq k$ and let $\rho(x)$ be the unique solution to the TF equation (6). Then

$$
\rho^{+}(x)>\rho^{-}(x), \quad \text { all } x \in P^{+}(n, d) .
$$

Lemma 5. Let $n$ and $d$ be as in Lemma 4. Let $V$ be the Coulomb potential (3). Then

$$
V^{+}(x)>V^{-}(x), \quad \text { all } x \in P^{+}(n, d) .
$$

Remark. This property of $V$ is exactly the one needed to prove Theorem 1 and 4 in [5] and Theorem 3.2 in [9].

We will now prove the analog of Theorem 1 for the regularized TF model:

Theorem 6. Fix $z_{i}$ all $1 \leqq i \leqq k$ and $\lambda \leqq Z$. Fix $j(1 \leqq j \leqq k)$ and assume that $R_{j}$ is such that

$$
R_{i} \in P^{+}\left(n, n \cdot R_{j}\right) \cup P\left(n, n \cdot R_{j}\right), \quad \text { all } i \neq j
$$

for some fixed unit vector $n \in \mathbb{R}^{3}$. Let $E_{a}(\lambda, \alpha) \equiv E_{a}\left(\lambda ;\left\{R_{1}, \ldots, R_{j-1}, R_{j}+\alpha n\right.\right.$, $\left.\left.R_{j+1}, \ldots, R_{k}\right\}\right)$ and $e_{a}(\lambda ; \alpha) \equiv e_{a}\left(\lambda ;\left\{R_{1}, \ldots, R_{j-1}, R_{j}+\alpha n, R_{j+1}, \ldots, R_{k}\right\}\right)$. Choose $a<\min _{i}\left\{\operatorname{dist}\left(R_{i}, P\left(n, n \cdot R_{j}\right)\right) \mid R_{i} \notin P\left(n, n \cdot R_{j}\right)\right\}$. Then,

(i) $E_{a}(\lambda ; \alpha)$ is a monotonic non-increasing function of $\alpha \geqq 0$,

(ii) $e_{a}(\lambda ; \alpha)$ is a monotonic non-decreasing function of $\alpha \geqq 0$,

(iii) for fixed $\alpha>0, E_{a}(\lambda ; \alpha)-E_{a}(\lambda ; 0)$ is non-decreasing in $\lambda$,

(iv) for fixed $\alpha>0, e_{a}(\lambda ; \alpha)-e_{a}(\lambda ; 0)$ is non-decreasing in $\lambda$.

Proof. (i) By the Feynman-Hellman Theorem 2 [see Sect. 2, Eq. (17)],

$$
\left.\frac{d E_{a}}{d \alpha}\right|_{\alpha=0}=-z_{j} \int\left[n \cdot \nabla g_{a}\left(y-R_{j}\right)\right] \phi_{a}(y) d y .
$$

We can write the right side of (24) as

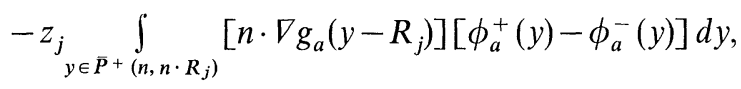

because $n \cdot \nabla g_{a}\left(y-R_{j}\right)$ only changes sign under a reflection (of $y$ ) through $P\left(n, n \cdot R_{j}\right)$. Here $\phi_{a}^{ \pm}$are defined as in (23) with respect to the plane $P\left(n, n \cdot R_{j}\right)$. Since $n \cdot \nabla g_{a}\left(y-R_{j}\right) \geqq 0$, all $y \in P^{+} \cup P$ [because $g_{a}(y)$ is spherically symmetric and decreasing] and $\phi_{a}^{+}(y) \geqq \phi_{a}^{-}(y)$, all $y \in P^{+} \cup P$ [Lemma 3, (i)], (25) is nonpostive and (i) follows.

(iii) Write $E_{a}(\lambda, \alpha)-E_{a}(\lambda, 0)=\int_{0}^{\alpha} d E_{a} / d \alpha d \alpha$. Now, for every fixed $\alpha, d E_{a} / d \alpha(\lambda, \alpha)$ is an increasing function of $\lambda$ because of (25) and Lemma 3(ii). 
(ii) By integration by parts one can write (15) as

with

$$
\left.\frac{d e_{a}}{d \alpha}\right|_{\alpha=0}=z_{j} \int p_{j}(x) \rho_{a}(x) d x
$$

$$
p_{j}(x) \equiv n \cdot \nabla_{x} \int g_{a}(x-y)\left|y-R_{j}\right|^{-1} d y
$$

As before, we have

$$
\left.\frac{d e_{a}}{d \alpha}\right|_{\alpha=0}=z_{j} \int_{x \in \bar{P}^{+}} p_{\left(n, n \cdot R_{j}\right)} p_{j}(x)\left(\rho_{a}^{+}(x)-\rho_{a}^{-}(x)\right) d x,
$$

because $p_{j}(x)$ only changes sign under a reflection (of $x$ ) through $P\left(n, n \cdot R_{j}\right.$ ). Moreover, $p_{j}(x) \geqq 0$, all $x \in P^{+} \cup P$ (because $g_{a} *\left|x-R_{j}\right|^{-1}$ is spherically symmetric and decreasing). Hence, (ii) follows from (26) and Lemma 3(iii). Finally, it should be clear that (iii) is equivalent to (iv).

After all these preliminaries we can go to the

Proof of Theorem 1. Because of Lemma A.4, $V_{a} \rightarrow V$ as a $a$, in $L^{5 / 2}+L^{4}$. Therefore, Theorem II.15 in [1] implies that, for fixed $\lambda, e_{a}\left(\lambda ;\left\{R_{i}\right\}\right)$ $\rightarrow e\left(\lambda ;\left\{R_{i}\right\}\right)$ as $a \downarrow 0$. Hence Theorem 1 (ii) and (iv) follow from Theorem 6 (ii), (iv). Now, by Lemma A.5, $U_{a} \rightarrow U$ as $a \downarrow 0$, hence for fixed $\lambda$, $E_{a}\left(\lambda ;\left\{R_{i}\right\}\right) \rightarrow E\left(\lambda ;\left\{R_{i}\right\}\right)$ as $a \downarrow 0$. Therefore Theorem 1 (i) and (iii) follow from Theorem 6 (i), (iii).

\section{Proof of Theorem 1 (ii) for the Electronic Contribution to the Born-Oppenheimer Energy}

The electronic contribution to the Born-Oppenheimer energy for a system of one electron and $k$ (fixed) nuclei (located at $R_{i} \in \mathbb{R}^{3}$, with positive charge $z_{i}$ ) is defined by [5]:

with

$$
e\left(\left\{R_{i}\right\}\right)=\inf \operatorname{spec}\left(H_{e}\right)
$$

$$
H_{e}=-\Delta-\sum_{i=1}^{k} z_{i}\left|x-R_{i}\right|^{-1} \text {. }
$$

The behavior of $e\left(\left\{R_{i}\right\}\right)$ as a function of the nuclear coordinates has been studied in [5] and [9]. In particular, it has been shown [5] that $e\left(\left\{R_{i}\right\}\right)$ is monotonic non-decreasing under the dilation $R_{i} \rightarrow \ell R_{i}(\ell>1)$, all $i$. Here we will prove the following related result:

Theorem 7. Fix $z_{i}$, all $1 \leqq i \leqq k$. Fix $j(1 \leqq j \leqq k)$ and assume that $R_{j}$ is such that

$$
R_{i} \in\left\{x \in \mathbb{R}^{3} \mid\left(x-R_{j}\right) \cdot n \leqq 0\right\}, \quad \text { all } i \neq j,
$$

for some fixed unit vector $n \in \mathbb{R}^{3}$. Then $e\left(\left\{R_{1}, \ldots, R_{j-1}, R_{j}+\alpha n, R_{j+1}, \ldots, R_{k}\right\}\right)$ is a monotonic non-decreasing function of $\alpha \geqq 0$.

Remark. This is a straightforward generalization of Hoffman-Ostenhof's (Theorem 3.2, [9]) and therefore we will give here only the necessary modifications to his proof. 
Proof. Because of Lemma 5 (Sect. 3) and the comparison Theorem 2.3 of [9], we have that $\psi^{+}(x) \geqq \psi^{-}(x)$, all $x \in P^{+}\left(n, n \cdot R_{j}\right)$. Here $\psi^{-}$is the reflection of the ground state $\psi$ of $H_{e}$ with respect to $P\left(n, n \cdot R_{j}\right)$ and $\psi^{+}=\psi$. Therefore, by the Feynman-Hellman theorem we have

$$
\begin{aligned}
\left.\frac{d e}{d \alpha}\right|_{\alpha=0} & =-z_{j} \int\left|x-R_{j}\right|^{-3}\left(x-R_{j}\right) \cdot n \psi^{2}(x) d x \\
& =-z_{j} \int_{x \in P^{+}}\left|x-R_{j}\right|^{-3}\left(x-R_{j}\right) \\
& \geqq 0 . \quad \square
\end{aligned}
$$

\section{Appendix}

Let $f \in\left(L^{5 / 2}+L^{4}\right)\left(\mathbb{R}^{3}, d x\right)$ and define $f_{a}(x)=\left(g_{a} * f\right)(x)$, with $g_{a}$ given as in Sect. 2, for $a>0$. Since $f \in L_{\text {loc }}^{1}$, then $f_{a} \in C^{\infty}\left(\mathbb{R}^{3}\right)$ ([10], Lemma 2.18(a)). Moreover $f_{a} \in L^{5 / 2}+L^{4}$, by using Young's inequality.

Lemma A.1. (Continuity of $f_{a}(x)$ under translation.) Let $f_{a}^{\alpha}(x) \equiv f_{a}(x-\alpha R), \alpha>0$, $R \in \mathbb{R}^{3}$. Then $f_{a}^{\alpha} \rightarrow f_{a}$ in $L^{5 / 2}+L^{4}$ as $\alpha \downarrow 0$.

Proof. Since $\left(f_{a}^{\alpha}-f_{a}\right)(x)=\int\left[g_{a}(y-\alpha R)-g_{a}(y)\right] f(x-y) d y$, and $g_{a} \in C^{\infty}$, we have

$$
\begin{aligned}
\left|\left(f_{a}^{\alpha}-f_{a}\right)(x)\right| & \leqq \int d y|f(x-y)| \int_{0}^{1} d t \alpha\left|R \cdot \nabla g_{a}(y-t \alpha R)\right| \\
& \leqq \alpha\left\{\int d y|f(x-y)||R| \sup _{|z| \leqq \alpha|R|}\left|\nabla g_{a}(y-z)\right|\right\} .
\end{aligned}
$$

Now, $f \in L^{5 / 2}+L^{4}$ and $\nabla g_{a} \in L^{p}$ for any $1 \leqq p \leqq \infty$ hence $f_{a}^{\alpha} \rightarrow f_{a}$ in $L^{5 / 2}+L^{4}$ as $\alpha \downarrow$, by using Young's inequality.

Lemma A.2. $\alpha^{-1}\left(f_{a}^{\alpha}-f_{a}\right) \rightarrow \partial f_{a} /\left.\partial \alpha\right|_{\alpha=0}$ in $L^{5 / 2}+L^{4}$ as $\alpha \downarrow 0$.

Proof. We need only remark that for a $C^{2}(\mathbb{R})$ function $h$, we have

$$
b^{-1}[h(a+b)-h(a)]-h^{\prime}(a)=b \int_{0}^{1} d s \int_{0}^{s} d t h^{\prime \prime}(a+b t),
$$

for any $a, b \neq 0$. The rest of the proof proceeds as in the proof of Lemma A.1.

Lemma A.3. $U_{a}\left(\left\{R_{i}\right\}^{\alpha}\right)$ (given by Eq. (12) with $R_{1}$ replaced by $R_{1}+\alpha n, \alpha>0$, $n \in \mathbb{R}^{3}$ ) is continuously differentiable with respect to $\alpha$ and

$$
\frac{\partial U_{a}\left(\left\{R_{i}\right\}^{\alpha}\right)}{\partial \alpha}=-z_{1} \int\left[n \cdot \nabla g_{a}(y)\right] V_{a}^{\alpha}\left(y+R_{1}+\alpha n\right) d y .
$$

Proof. We need only prove differentiability at $\alpha=0$. From (12) we get

$$
\begin{aligned}
\alpha^{-1} & {\left[U_{a}\left(\left\{R_{i}\right\}^{\alpha}\right)-U_{a}\left(\left\{R_{i}\right\}\right)\right] } \\
& =z_{1} \sum_{j=2}^{k} z_{j} \int g_{a}(y) g_{a}(x) \alpha^{-1}\left[\left|x-R_{1}-\alpha n-y+R_{j}\right|^{-1}-\left|x-R_{1}-y+R_{j}\right|^{-1}\right] d x d y .
\end{aligned}
$$


Since $|x|^{-1} \in L^{5 / 2}+L^{4}$, Lemma A.2 implies that $\left.\alpha^{-1}\left(g_{a} *\left|x-R_{1}-\alpha n+R_{j}\right|^{-1}-g_{a} *\left|x-R_{1}+R_{j}\right|^{-1}\right) \rightarrow g_{a} * \frac{\partial}{\partial \alpha}\left|x-R_{1}-\alpha n+R_{j}\right|\right|_{\alpha=0}$ as $\alpha \downarrow 0$ in $L^{5 / 2}+L^{4}$. Since $g_{a} \in L^{p}$, any $1 \leqq p \leqq \infty$, and using the definition of $V_{a}$ [see Eq. (10)] we have

$$
\lim _{\alpha \downarrow 0} \alpha^{-1}\left[U_{a}\left(\left\{R_{i}\right\}^{\alpha}\right)-U_{a}\left(\left\{R_{i}\right\}\right)\right]=\left.z_{1} \int g_{a}(y) \frac{\partial V_{a}^{\alpha}}{\partial \alpha}\right|_{\alpha=0}\left(y+R_{1}\right) d y .
$$

[Notice that we have used $\int g_{a}(y) \partial /\left.\partial \alpha\left(|y-\alpha n|^{-1}\right)\right|_{\alpha=0} d y=0$ to obtain A.2.] Finally, (A.1) follows from (A.2) by integration by parts.

Lemma A.4. $f_{a} \rightarrow f$ in $L^{5 / 2}+L^{4}$ as $a \downarrow 0$.

Proof. See ([10], Lemma 2.18(c)).

Lemma A.5. $U_{a} \rightarrow U$ as $a \downarrow 0$.

Proof. We can write $U_{a}-U=\sum_{1 \leqq i<j \leqq k}\left(A_{i j}+B_{i j}\right)$ with

and

$$
A_{i j}=\int d x g_{a}(x)\left\{\int g_{a}(y)\left|x-y+R_{i}-R_{j}\right|^{-1} d y-\left|x+R_{i}-R_{j}\right|^{-1}\right\}
$$

$$
B_{i j}=\int d x g_{a}(x)\left|x+R_{i}-R_{j}\right|^{-1}-\left|R_{i}-R_{j}\right|^{-1} .
$$

Because of $\left([10]\right.$, Lemma 2.18(e)) $\lim _{a \downarrow 0} g_{a} *\left|x+R_{i}-R_{j}\right|^{-1}=\left|x+R_{i}-R_{j}\right|^{-1}$ uniformly on $|x| \leqq a$ (as long as $a<\left|R_{i}-R_{j}\right|$ ). Hence, $A_{i j} \rightarrow 0$ as $a \downarrow 0$. Also, $B_{i j} \rightarrow 0$ as $a \downarrow 0$ because of ([10], Lemma 2.18(e)).

Acknowledgements. I would like to thank Bradley Plohr for stimulating discussions and Elliot Lieb for useful discussions and encouragement.

\section{References}

1. Lieb, E.H., Simon, B.: Advan. Math. 23, 22-116 (1977)

2. Teller, E.: Rev. Mod. Phys. 34, 627-631 (1962)

3. Benguria, R., Lieb, E.H.: Commun. Math. Phys. 63, 193-218 (1978)

4. Narnhofer, H., Thirring, W.: Acta Phys. Aust. 41, 281-297 (1975)

5. Lieb, E.H., Simon, B.: J. Phys. B11, L 537-542 (1978)

6. Lieb, E.H., Brezis, H.: Commun. Math. Phys. 65, 231-246 (1979)

7. Lieb, E.H.: Thomas-Fermi and related theories of atoms and molecules. In: Proc. of the Conference on Rigorous Atomic and Molecular Physics, Erice, June, 1980

8. Brezis, H.: Some variational problems of the Thomas-Fermi type. In: Proc. Symp. Erice. New York: J. Wiley 1978

9. Hoffmann-Ostenhof, T.: J. Phys. A 13, 417-424 (1980)

10. Adams, R.A.: Sobolev spaces. New York: Academic Press 1975

Communicated by E. Lieb

Received December 12, 1980, in revised form March 30, 1981 